Molecular Physics, 1984, Vol. 52, No. 5, 1105-1113

\title{
Pressure dependence of the self-diffusion in liquid trifluoromethane
}

\author{
by F. X. PRIELMEIER, E. W. LANG and H.-D. LÜDEMANN \\ Institut für Biophysik und Physikalische Biochemie, \\ Universität Regensburg, Postfach 397, \\ D-8400 Regensburg, F.R. Germany
}

(Received 23 February 1984 ; accepted 24 March 1984)

\begin{abstract}
A simple modification of a high resolution N.M.R. spectrometer is presented, permitting determination of self-diffusion coefficients at pressures up to $200 \mathrm{MPa}$ in the temperature range between $140 \mathrm{~K}$ and $450 \mathrm{~K}$. The self-diffusion coefficients of $\mathrm{CHF}_{3}$ are determined between $142 \mathrm{~K}$ and $250 \mathrm{~K}$. The activation energies derived from the data are $\left[E_{\mathrm{a}} \mathrm{D}\right]_{p=\text { const }}=$ $(6.1 \pm 0.4) \mathrm{kJ} \mathrm{mol}^{-1}, \quad\left[E_{\mathrm{a}} \mathrm{D}\right]_{V=\text { const }}=(3.5 \pm 0.4) \mathrm{kJ} \mathrm{mol}^{-1}$. Except for the lowest temperatures, compressed $\mathrm{CHF}_{3}$ appears to behave as a normal, rough hard-sphere fluid.
\end{abstract}

\section{INTRODUCTION}

The single-particle dynamics of simple liquids can be derived from the investigation of the magnetic relaxation of the nuclear spins. However, the experiments give the spin-lattice- or spin-spin-relaxation rates at most at a few frequencies and one must thus rely on models or theories that connect the experimental data with the spectral density function. This fact is especially aggravating in low molecular weight, low viscosity liquids, since here the extreme narrowing limit condition applies and almost all relaxation mechanisms become independent of field and frequency $[1,2]$.

The self-diffusion coefficient $D$ is the only dynamic quantity, characterizing single-particle motion, that can be obtained directly from experiments without recourse to any model assumptions. Among the various methods used to determine the self-diffusion coefficient of a liquid, magnetic spin echo experiments in a steady or pulsed field gradient have become the method of choice. In the following a simple modification of a standard high resolution spectrometer is described, that permits the determination of self-diffusion coefficients between $150 \mathrm{~K}$ and $450 \mathrm{~K}$ and at pressures up to $200 \mathrm{MPa}$. In a first series of experiments with this apparatus the self-diffusion coefficient of liquid trifluoromethane has been determined. In recent theories of simple liquids [3-5] arguments have been put forward that in the absence of strong, anisotropic attractive interactions, like hydrogen bonds, the structure and dynamics of these liquids is dominated by short-ranged repulsive interactions. Thus the concept of the hard-sphere fluid as a zeroth order approximation to real liquids has been developed. Within this approximation, expressions for the self-diffusion coefficient have been established by forcing the density dependence to fit molecular dynamics calculations of the hard sphere fluid [6]. The self 


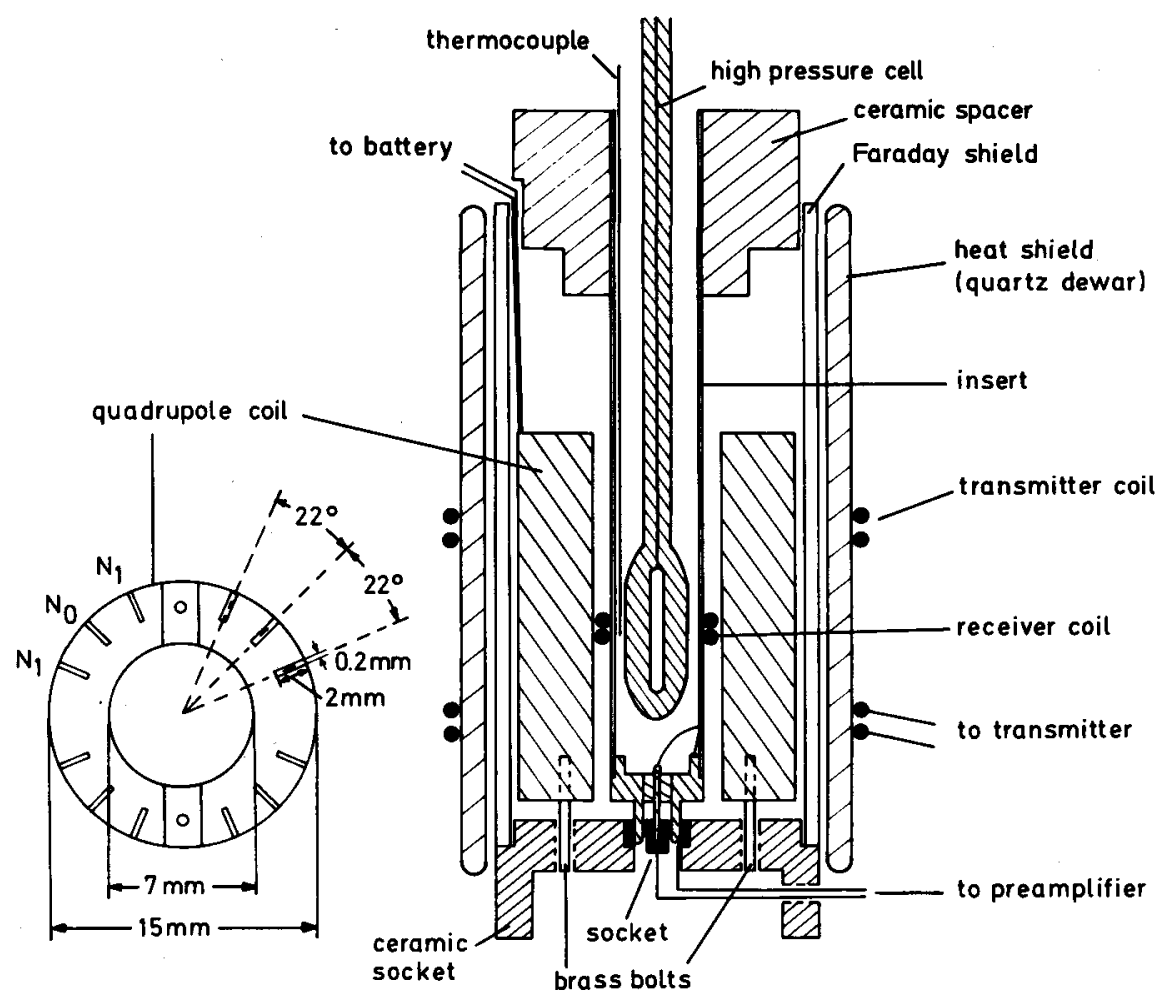

Figure 1. View of the variable-temperature, high-pressure device mounted in the standard Varian V 4415 probe. Left : Cross section of the gradient coil (Material : Macor glass ceramics).

diffusion coefficient of $\mathrm{CHF}_{3}$ as function of density are discussed within the framework of the hard sphere model for non-associated molecular liquids.

\section{EXPERIMENTAL}

The self-diffusion coefficients $D$ were determined in a Varian XL-100-15 FT spectrometer. Figure 1 gives a cross-section through the modified V-4415 probe. It was decided to use a quadrupole coil for the generation of the field gradients since this coil provides, compared with Helmholtz coils, larger horizontal gradients combined with a high gradient homogeneity along the vertical axis [7]. In addition it avoids magnetic-imaging problems and possesses a low inductivity only. The coil was fixed in the ceramic foot of the probe by two brass bolts. In order to minimize the effects of thermal expansion upon the coil geometry, the body of the coil was machined from glass ceramics (Macor, P. Schröder \& Co., 2086 Ellerau, F.R. Germany).

Each quadrant of the coil contains three groups of windings of insulated $0 \cdot 1 \mathrm{~mm}$ copper wire ( $N_{0}=14$ turns, $N_{1}=10$ turns). The current for the coil was provided by a battery and regulated with a potentiometer. It was controlled by a digital amperemeter with an accuracy of $\pm 0.05 \mathrm{~mA}$. The receiver coil is mounted on a thin-walled, ground quartz tube with an i.d. of $5.3 \mathrm{~mm}$. The limited space available within this insert prohibits the use of a burst protection 
around the high pressure glass cell. This cell was drawn from standard $5 \mathrm{~mm}$ o.d., $0.8 \mathrm{~mm}$ i.d. Duran 50 glass capillaries (Schott, Darmstadt, F.R. Germany). Details of the whole high pressure set-up, which is a modification of the strengthened glass-cell-design proposed by Yamada [8] have been given previously $[9,10]$.

The pressure was measured with a Bourdon gauge (Heise, Connecticut, U.S.A.) to $\pm 0.5 \mathrm{MPa}$. The temperature was regulated with the modified Varian variable temperatures accessory and controlled before and after each measurement by a miniature thermocouple (Philips, Kassel, F.R. Germany). The temperature could be kept constant to $\pm 0.5 \mathrm{~K}$ at any spot of the probe, however the introduction of the gradient coil partly obstructs the flow of the nitrogen gas used for thermostating and thus a small temperature gradient is established along the probe, leading to an overall temperature uncertainty of $\pm 1.5 \mathrm{~K}$. The spin echo was obtained with a $90^{\circ}-180^{\circ}$ pulse sequence. In the presence of a permanent field gradient the amplitude $M^{+}$of the echo is given by

$$
M^{+}(2 \tau)=M_{0} \exp \left[-2 \tau / T_{2}\right] \exp \left[-D(\gamma G)^{2} 2 \tau^{3} / 3\right],
$$

with $\tau$ the interval between the $90^{\circ}$ and $180^{\circ}$ pulse, $T_{2}$ the spin-spin-relaxation time, $D$ the self-diffusion coefficient, $\gamma$ the gyromagnetic ratio of the nucleus studied and $G$ the magnetic field gradient $(G=k I, k=$ coil constant). $D$ can be derived either from a series of echoes obtained at constant $G$ under variation of $\tau$ or at constant $\tau$ by applying an increasing gradient.

It appeared preferable to use the second alternative and work at constant $\tau$. The gradient $G$ was calibrated by determining the spin echoes of a neat oxygen free, water sample, for which the self-diffusion coefficient is well known [11]. The calibration was performed between $277 \mathrm{~K}$ and $368 \mathrm{~K}$. The coil constant $k$ is independent of temperature in this interval. During the course of the experiments several coils were applied for which $k$ varied from $0 \cdot 70 \pm 0 \cdot 01$ to $0.90 \pm 0.01\left(\mathrm{Tm}^{-1} \mathrm{~A}^{-1}\right)$. Since the coil constant depends on the exact alignment of the coil in the $B_{0}$-field, the calibration is regularly repeated, before any measurements are started.

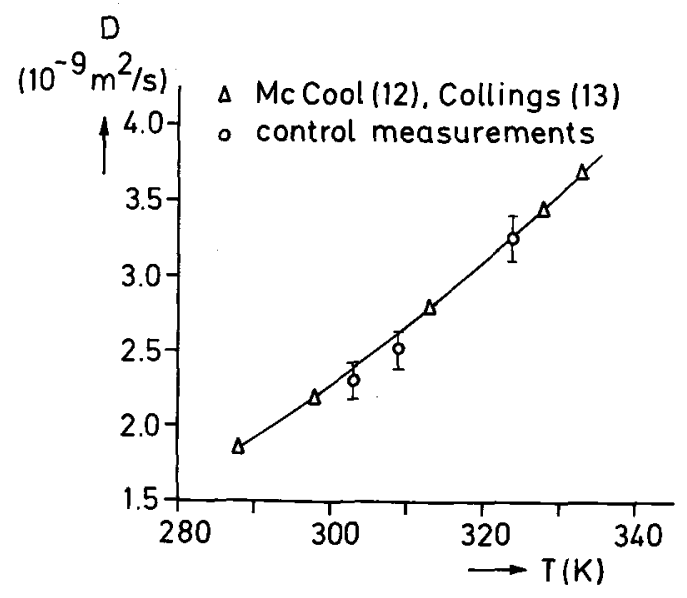

Figure 2. Comparison of the control measurements of the self-diffusion coefficient of liquid benzene with data from the literature. 
The spin echoes are accumulated in the memory of the computer and are rectified before plotting. The amplitude of the signal as function of the gradient coil current $A(\mathrm{I})$ is registered and from a plot of $\log A$ versus $I^{2}$ the self-diffusion coefficient $D$ is determined. The calibration of the set-up was controlled by measuring the ambient pressure self-diffusion coefficient of benzene. Figure 2 compares the published data $[12,13]$ for this substance with our experiments.

Trifluoromethane was purchased from Baker Chemikalien (6080 GroßGerau, F.R. Germany). It was dried over molecular sieve $3 \AA$ and stored in a 51 glass flask. Prior to use it was degassed by at least 5 freeze-pump-thawcycles to a final pressure of $5 \times 10^{-3} \mathrm{~Pa}$. Details of the filling procedure for the high pressure cell and the high vacuum apparatus used have been published previously [14]. All self-diffusion coefficients are judged reliable to \pm 10 per cent.

\section{RESULTS AND DISCUSSION}

The self-diffusion coefficients $D$ determined are compiled in table 1 . In the literature only a few self-diffusion coefficients of $\mathrm{CHF}_{3}$ at saturation pressure between $113 \mathrm{~K}$ and $173 \mathrm{~K}$ [15] could be found. These coefficients are approximately $2 \frac{1}{2}$ times larger than our results. This difference must remain unexplained, since the samples have been cleaned carefully in both cases, as can

Table 1. Self-diffusion coefficents $D\left(10^{-9} \mathrm{~m}^{2} \mathrm{~s}^{-1}\right)$ for liquid $\mathrm{CHF}_{\mathbf{3}}$.

\begin{tabular}{|c|c|c|c|c|c|c|c|c|c|}
\hline \multicolumn{10}{|c|}{ Saturation pressure } \\
\hline$T(\mathrm{~K})$ & 144 & 158 & 187 & 216 & 243 & & & & \\
\hline$D$ & $0 \cdot 88$ & 1.4 & 2.7 & 4.9 & $7 \cdot 1$ & & & & \\
\hline \multicolumn{10}{|c|}{$p=10 \mathrm{MPa}$} \\
\hline$T(\mathrm{~K})$ & 147 & 161 & 182 & 205 & 246 & & & & \\
\hline$D$ & 0.93 & 1.5 & $2 \cdot 4$ & 4.9 & $7 \cdot 4$ & & & & \\
\hline \multicolumn{10}{|c|}{$p=50 \mathrm{MPa}$} \\
\hline$T(\mathrm{~K})$ & 142 & 147 & 159 & 161 & 168 & 182 & 195 & 205 & 246 \\
\hline $\bar{D}$ & 0.72 & 0.81 & 1.1 & 1.1 & 1.5 & $2 \cdot 1$ & 2.7 & 3.7 & $6 \cdot 1$ \\
\hline \multicolumn{10}{|c|}{$p=100 \mathrm{MPa}$} \\
\hline$T(\mathrm{~K})$ & 142 & 147 & 160 & 161 & 168 & 205 & 224 & 243 & 246 \\
\hline$D$ & 0.52 & 0.61 & 0.78 & 0.86 & $1 \cdot 1$ & $2 \cdot 1$ & $2 \cdot 8$ & $3 \cdot 5$ & 4.9 \\
\hline \multicolumn{10}{|c|}{$p=150 \mathrm{MPa}$} \\
\hline$T(\mathrm{~K})$ & 144 & 166 & 173 & 184 & 194 & 204 & 250 & & \\
\hline$D$ & 0.44 & 0.84 & 0.93 & 1.4 & 1.5 & 1.9 & 4.0 & & \\
\hline \multicolumn{10}{|c|}{$p=200 \mathrm{MPa}$} \\
\hline$T(\mathrm{~K})$ & 166 & 184 & 204 & 255 & & & & & \\
\hline$D$ & 0.60 & 1.1 & 1.7 & $2 \cdot 2$ & & & & & \\
\hline
\end{tabular}




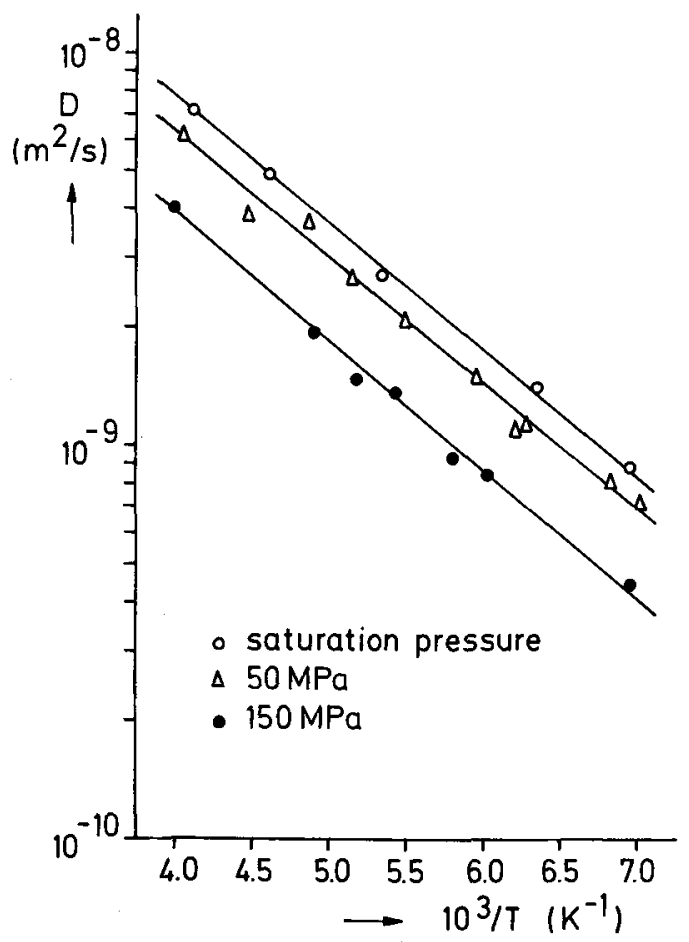

Figure 3. Temperature dependence of the self-diffusion coefficient of liquid $\mathrm{CHF}_{3}$ at saturation pressure and at 50 and $150 \mathrm{MPa}$.

be deduced from the identical ${ }^{19} \mathrm{~F}$ and ${ }^{1} \mathrm{H}$ spin lattice relaxation times $[15,22]$. Thus the only plausible explanation for the difference could be convection of the $\mathrm{CHF}_{3}$ caused by a temperature gradient or an error in the determination of the steady field gradient.

The isobars between saturation pressure and $200 \mathrm{MPa}$ are within the limits of error straight lines with identical slopes. Figure 3 gives three of these isobars. The data at intermediate pressures have been omitted from the graph for the sake of clarity. A compact and convenient way to express experimental results is to calculate activation energies $\Delta E^{\neq}$and activation volumes $\Delta V^{\ddagger}$ as a means to compare the pressure and temperature dependence of dynamic properties of different systems. This analysis is meaningful as long as an Arrhenius behaviour is found for the property under consideration although the underlying physical concept of an activated state may be questionable. From these isobars the activation energy at constant pressure can be calculated :

$$
\left(\Delta E^{\ddagger}\right)_{p=\text { const }}:=-R\left(\frac{\partial \ln D}{\partial \frac{1}{T}}\right)_{p}=(6 \cdot 1 \pm 0 \cdot 4) \mathrm{kJ} \mathrm{mol}^{-1}
$$

From the isobars five isotherms have been constructed. These are shown in figure 4. The data at $188 \mathrm{~K}, 208 \mathrm{~K}$ and $250 \mathrm{~K}$ do show a pronounced curvature, the slope decreasing with increasing pressure, while the isotherms at 


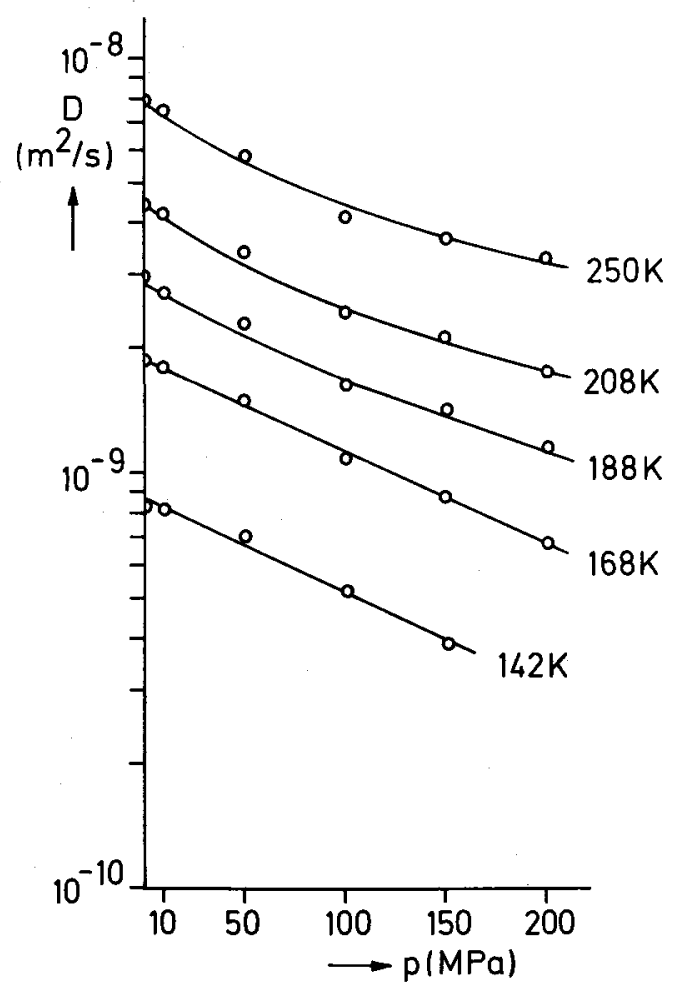

Figure 4. Pressure dependence of the self-diffusion coefficient $D$ of liquid $\mathrm{CHF}_{3}$.

Table 2. Activation volumes for the self-diffusion in $\mathrm{CHF}_{3}$ at saturation pressure and at $200 \mathrm{MPa}$.

\begin{tabular}{lccccc}
\hline \multicolumn{1}{c}{$T(\mathrm{~K})$} & 142 & 168 & 188 & 208 & 250 \\
\hline $\begin{array}{l}\Delta V_{\text {s.p. }} \\
\left(10^{-6} \mathrm{~m}^{3} \mathrm{~mol}^{-1}\right)\end{array}$ & & & $9 \cdot 2 \pm 1 \cdot 0$ & $13 \pm 1 \cdot 5$ & $18 \pm 2 \cdot 0$ \\
$\begin{array}{l}\Delta V_{200} \mathrm{MPa}^{-1} \\
\left(10^{-6} \mathrm{~m}^{3} \mathrm{~mol}^{-1}\right)\end{array}$ & $6 \cdot 2 \pm 1 \cdot 0$ & $7 \cdot 1 \pm 1 \cdot 0$ & & & \\
\end{tabular}

$142 \mathrm{~K}$ and $168 \mathrm{~K}$ are linear in the $\log D$ versus $p$ presentation. From the slopes of the isotherms (figure 4 ) the activation volume for self-diffusion is derived :

$$
\Delta V^{\ddagger}:=-R T\left(\frac{\partial \ln D}{\partial p}\right)_{T} .
$$

The data obtained at saturation pressure and at $200 \mathrm{MPa}$ are compiled in table 2.

The isotherms of the $\log D$ versus pressure representation of figure 4 yield only a qualitative impression about the density dependence of the self-diffusion coefficient in $\mathrm{CHF}_{3}$. For a separation of the effects of the thermal energy 
from the density variation one needs $p V T$ data of the compound. Experimental data for the temperature and pressure region covered by our experiments are lacking. Kruse [16] correlated existing data on $\mathrm{CHClF}_{2}$ and $\mathrm{CHF}_{3}[17,18]$ and gives extrapolated densities in the pressure range up to $300 \mathrm{MPa}$ at temperatures between $300 \mathrm{~K}$ and $500 \mathrm{~K}$. Gallant [19] gives data for the liquid density of $\mathrm{CHF}_{3}$ at saturation pressure down to $150 \mathrm{~K}$. Densities extrapolated graphically from the data given by Kruse [16] yield within \pm 2 per cent the same densities as given by Gallant [19]. We therefore consider our crudely interpolated densities reliable to \pm 4 per cent.

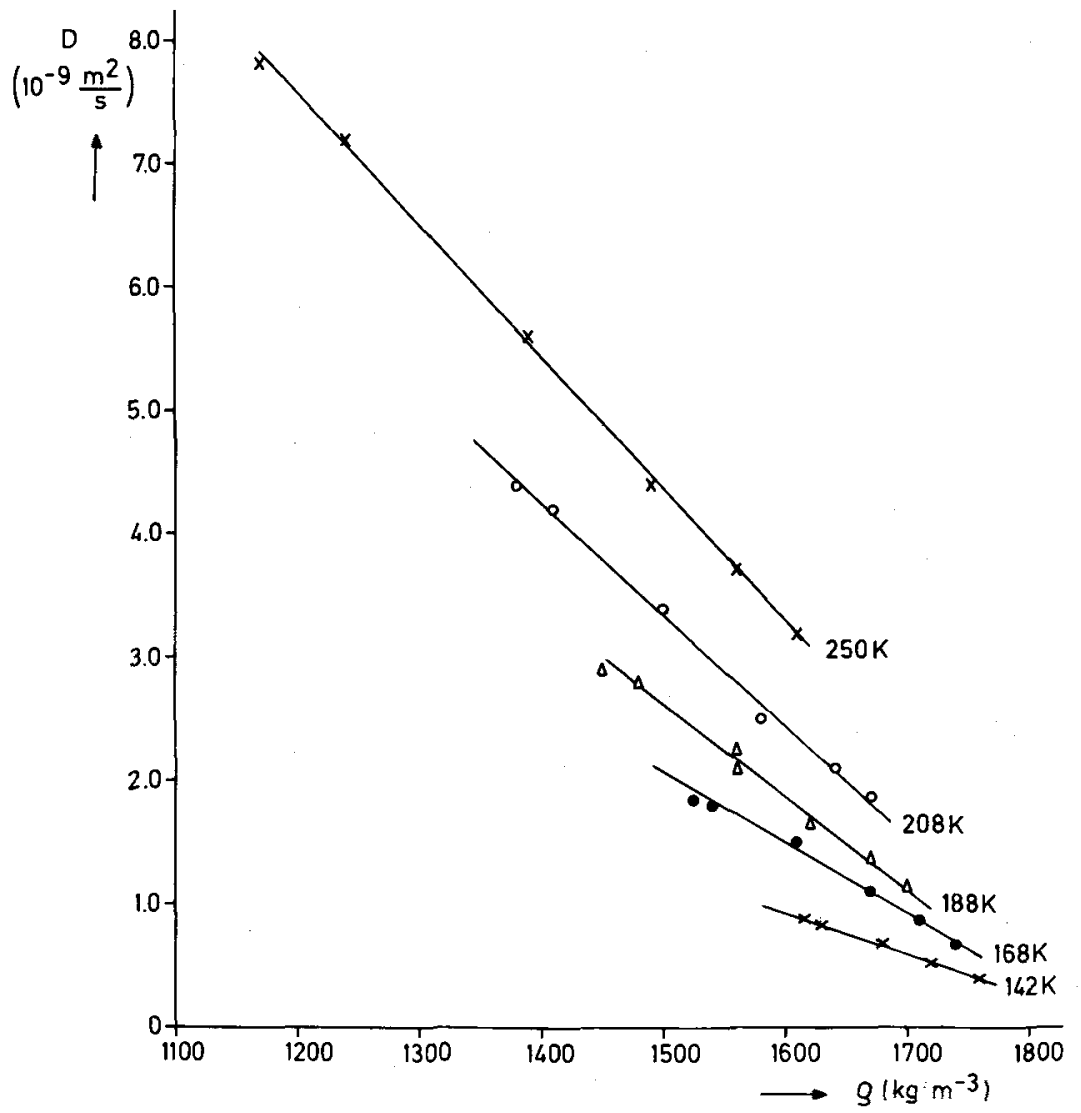

Figure 5. Density dependence of the self-diffusion coefficient $D$ of liquid $\mathrm{CHF}_{3}$.

Figure 5 contains the isothermal density dependence of the self-diffusion coefficient. The points given in the diagram are taken from figure 4 . A linear density dependence is found with a slope increasing with rising temperature. From an Arrhenius plot of the isochores for $1400 \leqslant \rho \leqslant 1700\left(\mathrm{~kg} \mathrm{~m}^{-3}\right)$, which are within the accuracy of the data linear with identical slopes, an activation energy at constant volume : $\left(E_{\mathrm{a}}^{\mathrm{D}}\right)_{V=\text { const }}=(3 \cdot 5 \pm 0 \cdot 4) \mathrm{kJ} \mathrm{mol}^{-1}$ is calculated. The ratio of the two activation energies $\left(E_{\mathrm{a}}\right)_{p} /\left(E_{\mathrm{a}}\right)_{V}$ at constant pressure and at constant volume is close to 0.5 indicating that liquid $\mathrm{CHF}_{3}$ behaves like a normal simple liquid [20]. For hydrogen bonded liquids like water or ammonia [21] these two activation energies are approximately the same. 
The dynamics of simple liquids, composed of nearly spherical molecules, interacting mainly via short-ranged repulsive forces, are often well described by the corrected Enskog [23] theory as introduced by Dymond [3]. The selfdiffusion coefficient of the smooth hard-sphere fluid $D_{\mathrm{shs}}$ is given in terms of the packing fraction $\rho d^{3}$ of the liquid as [3-5]

$$
D_{\mathrm{sh} 8}=\frac{3}{8}\left(\frac{k T}{m \pi}\right)^{1 / 2} d P\left(\rho d^{3}\right)
$$

with $m$ the molecular mass, $d$ the hard-sphere diameter, $\rho$ the number density and $P\left(\rho d^{3}\right)$ an empirical polynomial in the packing fraction $\rho d^{3}$ used to interpolate the molecular dynamics results of the hard-sphere fluid as obtained by Alder et al. [6]. In rough hard-sphere fluids or molecular liquids composed of particles with a non-spherical shape, rotation-translation coupling can occur with the effect of lowering the diffusion coefficient from what one would find in its absence. Chandler $[4,25,26]$ has argued that this phenomenon can be taken into account by setting

$$
D \simeq D_{\text {rhs }}=A D_{\text {shs }},
$$

where $D$ is the self-diffusion coefficient of the molecular liquid, $D_{\text {rhs }}$ and $D_{\text {shs }}$ is the diffusion coefficient of the rough and smooth hard sphere fluid at the same packing fraction $\rho d^{3}$ and the parameter $A$ measures the degree of rotationtranslation coupling. Dymond [3], Trappeniers [5] and Chandler [4] use different interpolation formulae to represent the density dependence of diffusion coefficients of the hard-sphere fluid. As the hard-sphere diameter is not known a priori, equation (2) is normally used together with the measured isothermal density dependence of the self-diffusion coefficient to estimate the hard-sphere diameter from the slope $(\partial \ln D / \partial \ln \rho)_{T}$ which can be compared to $\partial \ln D_{\text {sls }} /$ $\partial \ln \left(\rho d^{3}\right)$. Note that the latter quantity is a function of the packing fraction only. For the density range $0.55<\left(\rho d^{3}\right)<0.95$ covered by our experiments all three equations (3)-(5) yielded nearly identical hard-sphere diameters as calculated from the isotherms of figure 5. They are collected in table 3. They show a weak temperature dependence with a decreasing hard-sphere diameter with increasing temperature due to the higher kinetic energy of the particles. With the hard-sphere diameter thus obtained the coupling parameter $A$ can be obtained from equation (3). It is also given in table 3. A noticeable feature is the temperature dependence of the slope of $(\partial D / \partial \rho)_{T}$ which should be $\sim \sqrt{ } T$ as predicted by theory (see (3)). In most liquids, however, a stronger

Table 3. Hard-sphere diameters $d$ and $A$-parameter $\left(A=D_{\text {sns }} D_{\exp ^{-1}}\right)$ of liquid $\mathrm{CHF}_{3}$ derived from the data given in figure 4.

\begin{tabular}{ccc}
\hline$T(\mathrm{~K})$ & $d(\mathrm{~nm})$ & $A$ \\
\hline 142 & 0.396 & 0.38 \\
168 & 0.398 & 0.57 \\
188 & 0.399 & 0.65 \\
208 & 0.390 & 0.60 \\
250 & 0.378 & 0.59 \\
\hline
\end{tabular}


increase with temperature of the slope in the $D$ versus $\rho$ plot is found. This has been interpreted to mean that the rotation-translation coupling factor is temperature dependent [24]. Considering the experimental errors no variation of $A$ with temperature is seen except for the lowest temperature of $142 \mathrm{~K}$. At the higher temperatures, the coupling parameter $A$ is close to the value found for carbontetrachloride [4] $(A=0.54)$, which appears to be a typical example of a rough hard sphere fluid. The decrease at $142 \mathrm{~K}$ could indicate, that at low temperatures the dipole moment of $\mathrm{CHF}_{3}$ of $1.65 \mathrm{D}$ leads to more effective coupling of translation and rotation of the single molecule and thus to a temperature dependent coupling factor. However, the deviation can also be ascribed to the influence of the attractive part of the intermolecular potential at lower temperatures $[27,28]$.

The expert technical assistance by G. Wührl, R. Knott and S. Heyn made these experiments possible. Their contributions are gratefully acknowledged. Financial support for this work was given by the DFG and the Fonds der Chemie.

\section{REFERENCES}

[1] Abragam, A., 1961, The Principles of Nuclear Magnetism (Oxford University Press).

[2] SpIEss, H. W., 1978, NMR-Basic Principles and Progress, Vol. 15, edited by P. Diehl, E. Fluck and R. Kosfeld (Springer-Verlag), pp. 55ff.

[3] Dymond, J. H., 1974, f. chem. Phys., 60, 969.

[4] Chandler, D., 1975, $\mathcal{Y}$. chem. Phys., 62, 1358.

[5] Trappeniers, N. J., 1982, Adv. molec. Relax. Interaction Processes, 24, 297.

[6] Alder, B. J., Gass, D. M., and Wainwright, T. E., 1970, f. chem. Phys., 53, 3813.

[7] Webster, D. S., and Marsden, K. H., 1974, Rev. scient. Instrum., 45, 1232.

[8] Yamada, H., 1974, Rev. scient. Instrum., 45, 690.

[9] GaArz, U., and Lüdemann, H.-D., 1976, Ber. Bunsenges. phys. Chem., 80, 607.

[10] Völkel, G., Lang, E., Lüdemann, H.-D., 1979, Ber. Bunsenges. phys. Chem., 83, 722.

[11] Weingärtner, H., 1982, Z. phys. Chem., 132, 129.

[12] McCool, M. A., Collings, A. F., and Woolf, L. A., 1972, J. chem. Soc. Faraday I, 68, 1489.

[13] Collings, A. F., and Woolf, L. A., 1975, Y. chem. Soc. Faraday I, 71, 2296.

[14] Hauer, H., Lang, E. W., and Lüdemann, H.-D., 1979, Ber. Bunsenges. phys. Chem., $83,1262$.

[15] Chaffin, J. H., and Hubbard, P. S., 1967, f. chem. Phys., 46, 1511.

[16] Kruse, R. A., 1981, Dissertation, Karlsruhe.

[17] Hou, Y. C., and Martin, J. J., 1959, A.I.Ch.E. Fl, 5, 125.

[18] WAGNER, W., 1968, Kältetechnik, 20, 238.

[19] Gallant, R. W., 1968, Hydrocarbon Process., 47, 89.

[20] Wilbur, D. J., and Jonas, J., 1975, F. chem. Phys., 62, 2800. Jonas, J., DeFries, T., and Wilbur, D. J., 1976, F. chem. Phys., 65, 583.

[21] Hauer, H., Lang, E., and Lüdemann, H.-D., 1979, Ber. Bunsenges. phys. Chem., 83, 1262. Lang, E. W., and Lüdemann, H.-D., 1982, Angerw. Chem., 94, 351 ; 1982, Int. Edn., 21, 315.

[22] Radkowitsch, H., 1983, Diplomarbeit Regensburg.

[23] Enskog, D., 1922, Kungl. svenska Vetensk-Akad. Handl., 63, 4.

[24] Parkhurst, H. J., and Jonas, J., 1975, J. chem. Phys., 63, 2698.

[25] Chandler, D., 1974, f. chem. Phys., 60, 3500.

[26] Chandler, D., 1974, F. chem. Phys., 60, 3508.

[27] Alley, W. E., and Alder, B. J., 1975, F. chem. Phys., 63, 3764.

[28] Kushick, J., and Berne, B. J., 1973, F. chem. Phys., 59, 3732. 